\title{
A Stable Aluminosilicate Zeolite with Intersecting Three-Dimensional Extra- Large Pores
}

Qing-Fang $\operatorname{Lin}^{1} \dagger$, Zihao Rei Gao ${ }^{2,3} \dagger$, Cong $\operatorname{Lin}^{4,5} \dagger$, Siyao Zhang ${ }^{1}$, Junfeng Chen ${ }^{6}$, Zhiqiang $\mathrm{Li}^{6}$, Xiaolong Liu', Wei Fan ${ }^{8}$, Jian $\mathrm{Li}^{2,4,9 *} \neq$, Xiaobo Chen ${ }^{6 *}$, Miguel A. Camblor ${ }^{3 *}$, Fei-Jian Chen ${ }^{1 *}$

\section{Affiliations:}

${ }^{1}$ Department of Chemistry, Bengbu Medical College; Bengbu 233030, China.

${ }^{2}$ Anhui ZEO New Material Technology Co., Ltd; 778 Dongliu Road, Hefei, 230071, China.

${ }^{3}$ Instituto de Ciencia de Materiales de Madrid, Consejo Superior de Investigaciones Científicas (ICMM-CSIC); c/Sor Juana Inés de la Cruz 3, Madrid 28049, Spain.

${ }^{4}$ College of Chemistry and Molecular Engineering, Peking University; Beijing, China.

${ }^{5}$ Department of Mechanical Engineering, The Hong Kong Polytechnic University; Kowloon, Hong Kong, China.

${ }^{6}$ State Key Laboratory of Heavy Oil Processing, China University of Petroleum; Qingdao 266580, China.

${ }^{7}$ State Key Laboratory of Optoelectronic Materials and Technologies, School of Materials, Sun Yat-Sen University; Guangzhou 510275, China.

${ }^{8}$ Department of Chemical Engineering, University of Massachusetts; Amherst, Massachusetts 01003, USA.

${ }^{9}$ Berzelii Center EXSELENT on Porous Materials, Department of Materials and Environmental Chemistry, Stockholm University; Stockholm, 10691, Sweden.

*Corresponding author. Email: cfj-cumt@163.com (F.-J.C.); macamblor@icmm.csic.es (M.A.C.); chenxiaobo@upc.edu.cn (X.C.); jxpxlijian@pku.edu.cn (J.L.).

†These authors contributed equally to this work.

¥Current address: Department of Fibre and Polymer Technology School of Engineering Sciences in Chemistry, Biotechnology and Health KTH Royal Institute of Technology; Tekninkringen 56-58, Stockholm, SE-100 44, Sweden.

Abstract: Zeolites are crystalline porous materials with important industrial applications, including uses in catalytic and adsorption/separation processes. Access into and out of their inner confined space, where adsorption and reactions occur, is limited by their pore apertures. Stable multidimensional zeolites with larger pores able to process larger molecules are in demand in the fine chemical industry and the oil processing on which the world still relies for fuels. Currently known extra-large pore zeolites display poor stability and/or lack pore multidimensionality, limiting their usefulness. We report ZEO-1, a robust fully-connected aluminosilicate zeolite with mutually intersecting three-dimensional extra-large plus three-dimensional large pores. ZEO-1 is stable up to 1000 Celsius, has an extraordinary specific surface area (1000 square meters per gram), and shows potential as a catalytic cracking catalyst.

One-Sentence Summary: ZEO-1 is the first aluminosilicate zeolite with multidimensional pores up to $10 \times 10 \AA$ and interconnecting supercages. 
Main Text: Zeolites with multidimensional interconnected extra-large pores, i.e. pores limited by rings of more than $12 \mathrm{SiO}_{4}$ or $\mathrm{AlO}_{4}$ members (>12-membered rings, MR), are in demand to allow the processing of large molecules (1). In the last three decades, 28 types of zeolites with extra-large pores have been successfully synthesized (2), but several factors limit their applicability. First, most of these zeolites require $\mathrm{Ge}$ to be synthesized, which increases their cost and severely decreases their thermal and hydrothermal stability $(3,4)$. Second, many are "interrupted frameworks" rather than true zeolites. Such frameworks lack tetrahedra sharing all of their vertices, which can decrease robustness $(5,6)$ and contain dangling $\mathrm{OH}$ groups that typically reduce the pore space (7). The only reported zeolites with multidimensional extra-large pores (-ITV, -CLO, -IFU, -IRY, and -IFT) are all interrupted germanosilicates or gallium phosphates with intrinsically low stability (8). Among the stable fully connected aluminosilicate zeolites, none possess a multidimensional system of extra-large pores but at most large 12MR pores in topologies discovered decades ago ( ${ }^{*} \mathbf{B E A}, \mathbf{F A U}$, and EMT) (2). The recently reported aluminosilicates PST-32 and PST-2 with known SBS and SBS/SBT topologies, have an aluminosilicate composition that ensures a good thermal stability and their pore architecture similar to FAU allows them to compete with this successful catalyst (9). The long lack of further improvements in the development of more open stable zeolites might suggest that there could be some sort of fundamental limit to the porosity exhibited by aluminosilicates. We report ZEO-1 (zeolite number 1 of Anhui ZEO New Material Technology Co., Ltd), an aluminosilicate zeolite with a multidimensional system of interconnected extra-large pores. This aluminosilicate has a high silica content $(\mathrm{Si} / \mathrm{Al}=14.5)$ and a zeolitic, non-interrupted framework, with high thermal and hydrothermal stability. The pore system of ZEO-1 contains both extra-large three-dimensional (3D) 16MR and large 3D 12MR channels with high interconnectivity that results in three types of supercages with four windows of $16 \mathrm{MR}$ and/or 12MR. Two of these supercages are larger than those found in FAU, EMT, and PST-32 and PST-2 (see Table S1). These features make ZEO-1 one of the stable zeolites with lowest framework density and ultrahigh surface area. Its open framework with large cavities accessible through 16MR windows along the three perpendicular directions (constituting a 3D extra-large 16MR pore system) and the presence in its pores of active acid sites enable good heavy-oil conversion and selectivity to fuels/commodities (gasoline, diesel, and liquified petroleum gas, LPG) during fluid catalytic cracking (FCC) competing with the performance of the highly optimized 12MR zeolite USY (ultra-stable Y zeolite, FAU topology), the current workhorse of this reaction.

Tricyclohexylmethylphosphonium (TCyMP) was used as the organic structure-directing agent (OSDA) for ZEO-1 (Fig. S1), whose complex structure was determined from nanosized crystals $(<200 \mathrm{~nm}$ ) by electron diffraction (3D ED) and refined against synchrotron powder x-ray diffraction (SPXRD), revealing that ZEO-1 crystallized in a body-centered tetragonal unit cell ( $a=43.3056(4)$ $\AA$ and $c=25.0010(8) \AA$ ) with Laue symmetry $4 / \mathrm{mmm}$. The structure determination was a great challenge because of the small crystal size (50 to $200 \mathrm{~nm}$, Fig. S2), large unit cell, and weakly scattering elements. We applied low-dose illumination and fast data acquisition to collect 3D ED data with continuous rotation electron diffraction (cRED) to overcome the difficulties of small crystals and electron-beam damage on the structure determination. The systematic extinctions suggested that the space group was $I 4_{1} /$ amd (\#141). The reconstructed reciprocal lattice from a typical dataset is shown in Fig. 1, A to E.

The structure of ZEO-1 calcined at $600{ }^{\circ} \mathrm{C}$ ( $3 \mathrm{~h}$ ramp, $6 \mathrm{~h}$ plateau) was successfully solved from cRED data (95\% completeness with a resolution of $0.81 \AA$ and a merging residual factor $R_{\text {int }}$ value of 0.3559, Table S3) by ab initio method with SHELXLT software (10). The framework was refined anisotropically against the cRED data without any restraints, and the refinement converged to an unweighted residual factor $\left(R_{1}\right)$ value of 0.1468 and goodness of fit (GoF) of 0.947 (Table S3). Further Rietveld refinement of the calcined structure against SPXRD data was performed (Fig. 1F and Table S7) resulting in more precise bond lengths and angles (Tables S8 and S9). Additionally, Rietveld refinement of the as-made material (Fig. S15 and Tables S4-S6) revealed that the disordered OSDA molecules are located in three unique positions (Figs. S13 and S14), which are 
within the three supercages with four windows of $16 \times 16 \mathrm{MR}, 16 \times 12 \mathrm{MR}$ or $12 \times 12 \mathrm{MR}$, respectively, with $0.394,0.499$, and 0.495 occupancies. The total number of OSDA molecules obtained from the Rietveld refinement was 44.42 per unit cell, in good agreement with the composition found by chemical analysis $\left(\left[\mathrm{Si}_{583.71} \mathrm{Al}_{40.29} \mathrm{O}_{1248}\right] \mid\left(\mathrm{C}_{19} \mathrm{H}_{36} \mathrm{P}\right)_{45.77}\left(\mathrm{H}_{2} \mathrm{O}\right)_{63.16}\right.$, Table S2).
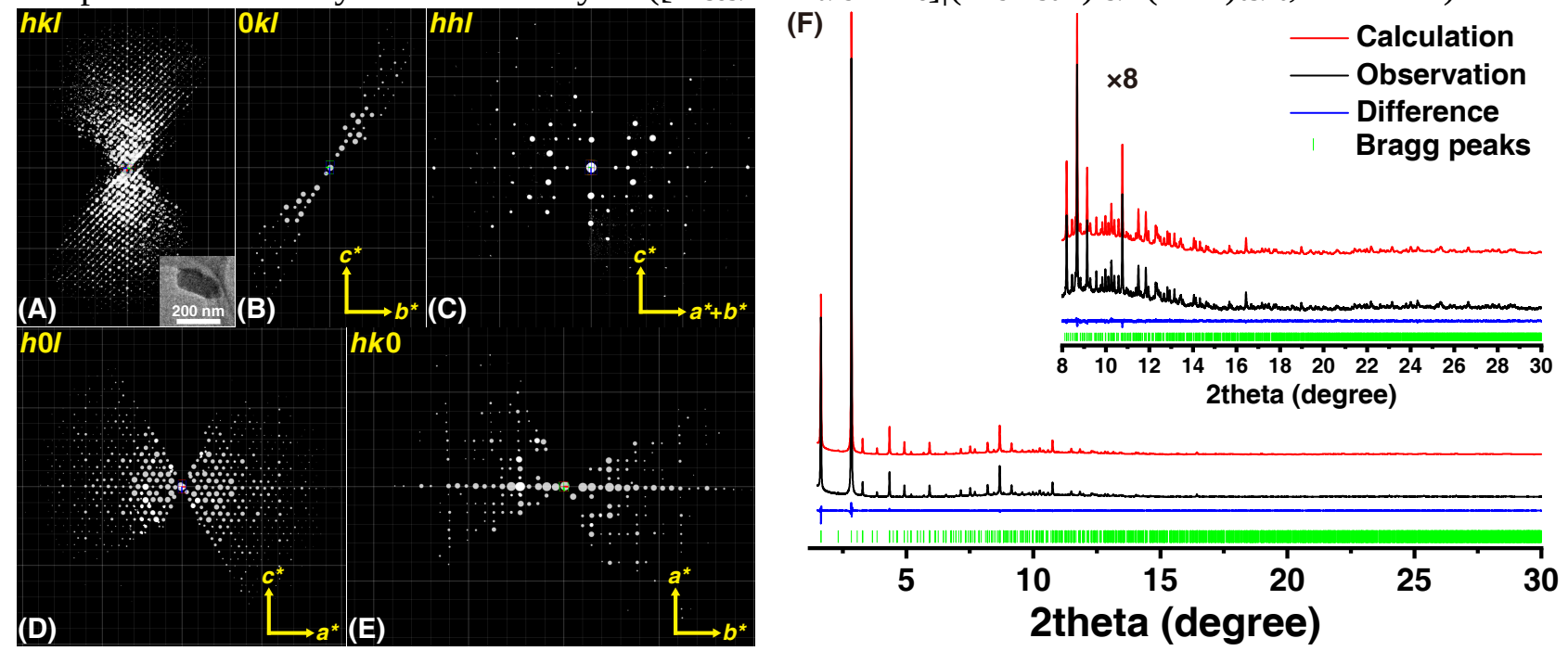

Fig. 1. Structure solution and Rietveld refinement of ZEO-1. (A) Projection of 3D reciprocal lattice reconstructed from a typical cRED dataset; the crystal (ZEO-1) from which data were collected is shown in the inset. The 2D slices of reflections $0 k l(\mathbf{B}), h h l(\mathbf{C}), h 0 l(\mathbf{D})$, and $h k 0(\mathbf{E})$ cut from the reconstructed reciprocal lattice. The reflection conditions are: $h k l: h+k+l=2 \mathrm{n} ; 0 \mathrm{kl}: k+l$ $=2 \mathrm{n} ; h h l: 2 h+l=4 \mathrm{n} ; h k 0: h=2 \mathrm{n}, k=2 \mathrm{n} ; 00 l: l=4 \mathrm{n}$, where $\mathrm{n}$ is a natural number. (F) Rietveld refinement plots of calcined ZEO- 1 , with $R_{p}=0.0279, R_{w p}=0.0337$, and $R_{\text {exp }}=0.0241$.

ZEO-1 has 21 unique T (tetrahedral) atoms and 43 unique oxygen atoms, which places it amongst the most complex fully connected zeolites solved to date. Only zeolites IMF, TUN, UOV, *STO, PST-20 (no code assigned so far) (11), and *SFV (12) with 24, 24, 25, 28, 29, and 99 unique T sites are more complex than ZEO-1. It also has a very large unit cell volume of $46900 \AA^{3}$, only surpassed by the higher members of the isoreticular embedded RHO family, i.e. RHO-G4 (ZSM-25, MWF) (8) and RHO-G5 (PST-20) (11). More complex members of this family of small pore zeolites with even larger volumes have not been solved so far (13). 


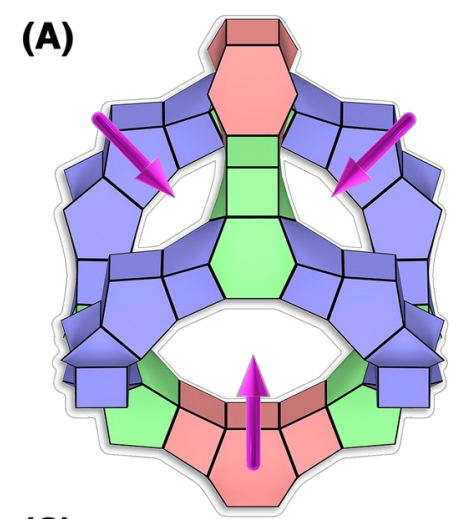

(C)

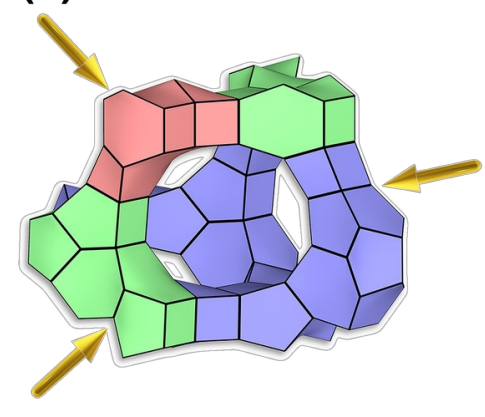

(B)

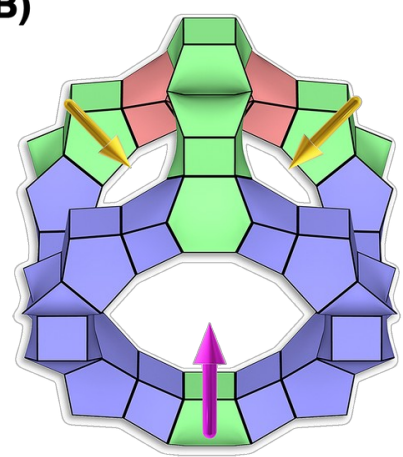

(D)

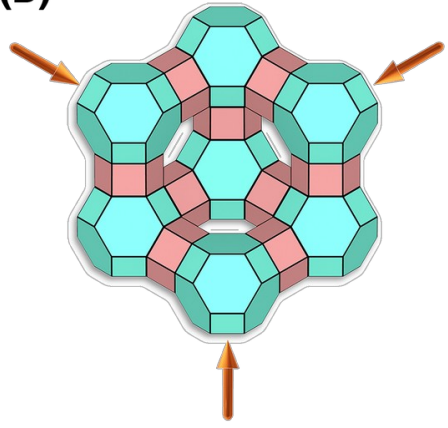

(E)
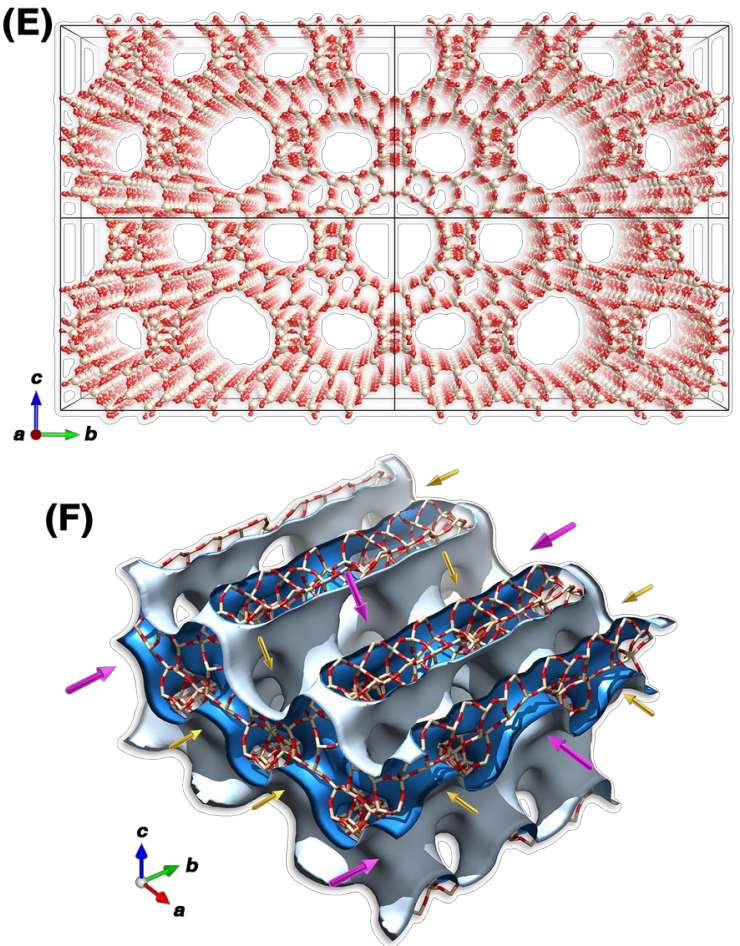

Fig. 2. The three supercages and channel system in ZEO-1. The pore system contains three supercages with 16MR (A), 16-12MR (B), and 12MR (C) apertures that are compared here to FAU 12MR supercage (D). (E) Structure viewed along $a$-axis and $(\mathbf{F})$ the $3 \mathrm{D}$ channel system with the structure model superimposed. Purple arrows: 16MR, gold arrows: 12MR; peach puff ball/stick: silicon, red ball/stick: oxygen.

Topological analysis indicates three different types of tiles occur at the channel intersections (Fig. 2, A to C). Those three tiles are the supercages with four windows of 16MR and/or 12MR, the ones containing 16MR being larger than the 12MR supercages in zeolites FAU ( $t$-fau, Fig. 2D), EMT ( $t$ wou), SBS (t-ucs and t-znf), and SBT (t-sbt) (Fig. S16). The channel system of ZEO-1 is 3D with interconnected $(16+12) \times(16+12) \times(16+12) \mathrm{MR}$ (Fig. 2, E and F), and the crystallographic pore apertures of the $16 \mathrm{MR}$ and $12 \mathrm{MR}$ are $10.62 \times 9.41 / 10.54 \times 9.64 \AA$ and $7.24 \times 6.60 / 7.18 \times 5.48 \AA$, respectively (Fig. S17).

Upon calcination in air to $600{ }^{\circ} \mathrm{C}$, the organic part of the OSDA within the ZEO- 1 channel could be removed while leaving behind phosphorous oxides (Fig. S3c) and the framework intact, as proved by PXRD (Fig. S8). The phosphorous species can be completely washed out with water (Fig. S3d). ZEO-1 is stable up to at least $1000{ }^{\circ} \mathrm{C}$ (Fig. S8). Calcined ZEO-1 exhibited type Ia $\mathrm{N}_{2}$ and $\mathrm{Ar}$ adsorption isotherms (Figs. S10 and S11), from which extraordinarily large specific surface area values of 939 and $1037 \mathrm{~m}^{2} / \mathrm{g}$, respectively, were determined by the Brunauer-Emmett-Teller (BET) method. From the Ar adsorption isotherm, the mean pore sizes of ZEO-1 were determined to be 6.7, 7.9, and $11.5 \AA$ (Fig. S12), which matched well with the crystallographic values (Fig. S17). The Horvath-Kawazoe pore-size distribution (Fig. S12) showed a maximum at $8.4 \AA$, indicating a substantially larger pore size than the reported values for the stable large pore faujasite (pore entrance $\sim 6.8 \AA$ ) and the recently reported PST-32 (SBS, $6.7 \AA$ ) and PST-2 (SBS/SBT intergrowth, $6.4 \AA$ ), all measured by Ar adsorption with the same formalism (9). Further, two of the supercages in ZEO-1 (those containing 16MR apertures, Fig. 2, A and B) are larger than the supercages in FAU, SBS, and SBT, while the third one (containing only 12MR apertures, Fig. 2C) is similar in size. ${ }^{29} \mathrm{Si}$ and ${ }^{27} \mathrm{Al}$ NMR (Figs. S5 and S6) show ZEO-1 contains framework Al that provides acidity in the calcined state, as determined by the $\mathrm{NH}_{3}$ adsorption and temperature-programmed desorption (Fig. S21) and $n$-decane cracking (Fig. S22). 
(A)

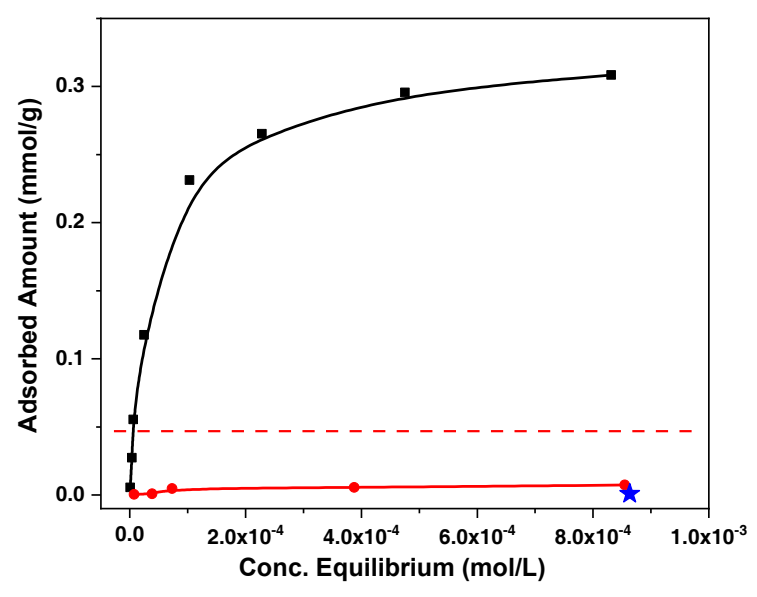

(B)

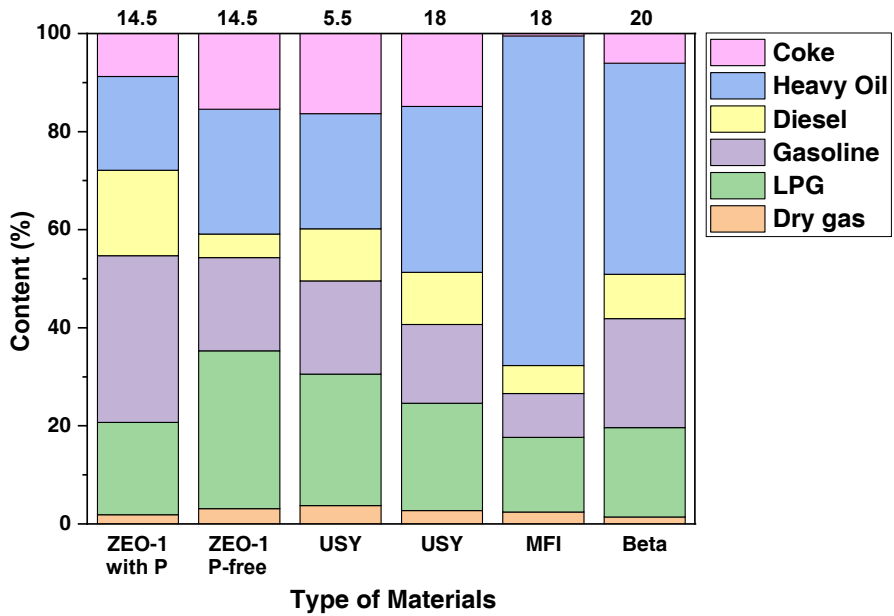

Fig. 3. The extra-large pores of ZEO-1 allow entrance/processing of large molecules. Nile Red adsorption isotherms (A, equilibrium concentration vs. adsorbed amount) at room temperature on ZEO-1 (black) and USY (red). The blue star corresponds to the adsorption on as-made ZEO-1, which reveals little external surface adsorption, while the dashed red line corresponds to reported values on FAU (14). Catalytic performance of several zeolite catalysts in the conversion of heavy oils $(\mathbf{B})$. Si/Al ratios are indicated on top.

The pores of ZEO-1 are larger than those of FAU (Fig. 2) and allow entrance of larger molecules, as shown by the adsorption of large dye molecules (Fig. S20). Although both ZEO-1 and USY readily adsorb methylene blue, ZEO-1 adsorbs $>40$ times more of the larger Nile Red than USY does at saturation (Fig. 3A). With regard to FCC, which converts heavy oil fractions into lighter, more valuable products (1), improved catalysts could increase the yield of fuels and commodities. Currently, zeolite Y (FAU) with 3D 12 MR channels and large supercages is the main active component in FCC catalysts (15).

Since phosphorous species can have an impact on the performance of zeolite catalysts (16), we performed FCC tests on ZEO-1 with and without washing out the occluded P species and on three commercial zeolite catalysts (USY, MFI, and Beta). These tests showed a beneficial effect of the occluded phosphorous as the unwashed ZEO-1 has the highest heavy-oil cracking conversion ratio (Fig. 3B, Tables S12 and S13), and the highest selectivity to the gasoline and diesel fuel fractions amongst all the catalysts. The washed catalyst shows a performance closer to the best USY catalyst tested, but with a larger selectivity to LPG. This change in selectivity from gasoline to LPG upon washing ZEO-1 is likely due to a highly branched gasoline fraction that is prone to cracking in this zeolite but not in the less acidic P-containing unwashed sample (Fig. S21) (17). These preliminary laboratory results show that ZEO-1 can compete with the highly optimized USY and also the recently reported PST-32 and PST-2 (9) and thus has a high potential as a component of cracking catalysts.

\section{References and Notes}

1. Reza Sadeghbeigi (2000). Fluid Catalytic Cracking Handbook (2nd ed.). Gulf Publishing. ISBN 0-88415-289-8

2. Ch. Baerlocher, L.B. McCusker, Database of Zeolite Structures: http://www.iza-structure.org/databases/.

3. L. Burel, N. Kasian, A. Tuel, Quasi All-Silica Zeolite Obtained by Isomorphous Degermanation of an As-Made Germanium-Containing Precursor. Angew. Chem. Int. Ed. 53, 1360-1363 (2014). doi:10.1002/anie.201306744. 
4. H. Xu, J. Jiang, B. Yang, L. Zhang, M. He, and P. Wu, Post-Synthesis Treatment gives Highly Stable Siliceous Zeolites through the Isomorphous Substitution of Silicon for Germanium in Germanosilicates. Angew. Chem. Int. Ed. 53, 1355-1359 (2014). doi:10.1002/anie.201306527.

5. Y. Chen, N. Ma, S. Huang, X. Du, F. Liao, Y. Zhu, J. Sun, Y. Wang, An Interrupted Zeolite PKU-26 and Its Transformation to a Fully Four-Connected Zeolite PKU-27 upon Calcination. Chem. Eur. J. 25, 3219-3223 (2019). doi:10.1002/chem.201805972.

6. Y. Wang, Y. Wang, J. Su, X. Song, W. Wan, J. Yu, Interrupted silicogermanate with 10-ring channels: synthesis and structure determination by combining rotation electron diffraction and powder X-ray diffraction. Inorg. Chem. Front. 4, 1654-1659 (2017). doi:10.1039/C7QI00309A.

7. R. Fricke, H.-L. Zubowa, M. Richter, H. Kosslick, Crystallization, stability and possible application of the molecular sieve cloverite. C. R. Chimie 8, 549-559 (2005).

doi:10.1016/j.crci.2004.09.010.

8. The structure commission of the International Zeolite Association (SC-IZA) assigns three letter Zeolite Framework Type codes to approved zeolite structures. Interrupted framework codes are preceded by a hyphen. See reference 2 .

9. H. Lee, J. Shin, K. Lee, H. J. Choi, A. Mayoral, N. Y. Kang, S. B. Hong, Synthesis of thermally stable SBT and SBS/SBT intergrowth zeolites. Science 373, 104-107 (2021). doi:10.1126/science.abi7208.

10. G. M. Sheldrick, SHELXT-Integrated space-group and crystal-structure determination. Acta Cryst. Sect. A 71, 3-8 (2015). doi:10.1107/S2053273314026370.

11. P. Guo, J. Shin, A. G. Greenaway, J. G. Min, J. Su, H. J. Choi, L. Liu, P. A. Cox, S. B. Hong, P. A. Wright, X. Zou, A zeolite family with expanding structural complexity and embedded isoreticular structures. Nature 524, 74-78 (2015). doi:10.1038/nature14575.

12. C. Baerlocher, T. Weber, L. B. McCusker, L. Palatinus, S. I. Zones, Unraveling the Perplexing Structure of the Zeolite SSZ-57. Science 333, 1134-1137 (2011). doi:10.1126/science.1207466.

13. J. Shin, H. Xu, S. Seo, P. Guo, J. G. Min, J. Cho, P. A. Wright, X. Zou, S. B. Hong, Targeted Synthesis of Two Super-Complex Zeolites with Embedded Isoreticular Structures. Angew. Chem. Int. Ed. 55, 4928-4932 (2016). doi:10.1002/anie.201510726.

14. I. Pellejero, M. Urbiztondo, D. Izquierdo, S. Irusta, I. Salinas, M. P. Pina, An Optochemical Humidity Sensor Based on Immobilized Nile Red in Y Zeolite, Ind. Eng. Chem. Res. 46(8), 2335-2341, (2007). doi:10.1021/ie061025v.

15. X. Li, C. Li, J. Zhang, C. Yang, H. Shan, Effects of Temperature and Catalyst to Oil Weight Ratio on the Catalytic Conversion of Heavy Oil to Propylene Using ZSM-5 and USY Catalysts, J. Nat. Gas Chem. 16, 92-99 (2007). doi:10.1016/S1003-9953(07)60033-4.

16. H. E. van der Bij, B. M. Weckhuysen, Phosphorus promotion and poisoning in zeolite-based materials: synthesis, haracterization and catalysis, Chem. Soc. Rev. 44, 7406-7428 (2015). doi:10.1039/C5CS00109A.

17. W. Vermeiren, J.-P. Gilson, Impact of Zeolites on the Petroleum and Petrochemical Industry, Top. Catal. 52, 1131-1161 (2009). doi:10.1007/s11244-009-9271-8.

18. T. Blasco, A. Corma, J. Martínez-Triguero, Hydrothermal stabilization of ZSM-5 catalyticcracking additives by phosphorus addition, J. Catal. 237, 267-277 (2006).

doi:10.1016/j.jcat.2005.11.011.

19. S. Smeets, B. Wang, stefsmeets/instamatic 1.6.0. Zenodo.

http://doi.org/10.5281/zenodo.4072976. 
20. W. Kabsch, Integration, scaling, space-group assignment and post-refinement. Acta. Cryst. Sect. D. 66, 133-144 (2010). doi:10.1107/S0907444909047374.

21. W. Wan, J. Sun, J. Su, S. Hovmoller, X. Zou, Three-dimensional rotation electron diffraction: software RED for automated data collection and data processing. J. Appl. Cryst. 46, 1863-1873 (2013). doi:10.1107/S0021889813027714.

22. O. V. Dolomanov, L. J. Bourhis, R. J. Gildea, J. A. K. Howard, H. Puschmann, OLEX2: A complete structure solution, refinement and analysis program. J. Appl. Cryst. 42, 339-341 (2009). doi:10.1107/S0021889808042726.

23. A. A. Coelho, TOPAS and TOPAS-Academic: an optimization program integrating computer algebra and crystallographic objects written in C++. J. Appl. Cryst. 51, 210-218 (2018). doi:10.1107/S1600576718000183.

24. S. Smeets, L. B. McCusker, C. Baerlocher, S. Elomari, D. Xie, S. I. Zones, Locating Organic Guests in Inorganic Host Materials from X-ray Powder Diffraction Data, J. Am. Chem. Soc. 138, 7099-7106 (2016). doi:10.1021/jacs.6b02953.

25. V. A. Blatov, A. P. Shevchenko, D. M. Proserpio, Applied Topological Analysis of Crystal Structures with the Program Package ToposPro, Cryst. Growth Des. 14(7), 3576-3586 (2014). doi:10.1021/cg500498k.

26. The GAVROG Project: http://gavrog.org/.

27. A. J. Aznar, B. Casal, E. Ruiz-Hitzky, I. Lopez-Arbeloa, F. Lopez-Arbeloa, J. Santaren, A. Alvarez, Adsorption of methylene blue on sepiolite gels: spectroscopic and rheological studies. Clay Minerals 27, 101-108 (1992). doi:10.1180/claymin.1992.027.1.10.

28. T. F. Willems, C. H. Rycroft, M. Kazi, J. C. Meza, M. Haranczyk, Algorithms and tools for high-throughput geometry-based analysis of crystalline porous materials, Microporous Mesoporous Mater., 149, 134-141 (2012). doi:10.1016/j.micromeso.2011.08.020.

\section{Acknowledgments:}

We are indebted to M. J. de la Mata (SIdI-UAM) for her expedited help in collecting the solidstate nuclear magnetic resonance spectra, and to the ALBA staff for collaboration in collecting the SPXRD data at the Spanish ALBA synchrotron beamline BL04 (MSPD).

Funding: We acknowledge financial support from the National Natural Science Foundation of China (grant numbers: 21601004, 21776312, 22078364), the Natural Science Foundation of the Higher Education Institutions of Anhui Province, China (grant numbers: KJ2020A0585), and the Spanish Ministry of Science Innovation and Universities (MICIU) (PID2019-105479RB-I00 project, AEI, Spain and FEDER, EU). The cRED data was collected at the Electron Microscopy Center (EMC), Department of Materials and Environmental Chemistry (MMK) in Stockholm University with the support of the Swedish Research Council (Grant No. 1444205) and the Knut and Alice Wallenberg Foundation (KAW, 2012-0112) through the 3DEM-NATUR project.. Use of the Advanced Photon Source at Argonne National Laboratory was supported by the U. S. Department of Energy, Office of Science, Office of Basic Energy Sciences, under Contract No. DE-AC02-06CH11357. W.F. gratefully acknowledges support from the U.S. Department of Energy, Office of Science, Basic Energy Sciences, Materials Sciences and Engineering Division, under Award \# DE-SC0019170.

Author contributions: F.-J.C. designed the project. J.L., X.C., M.A.C., and F.-J.C. supervised the work. Q.-F.L., S.Z., and F.-J.C. carried out the synthesis work. J.L. solved the structure. Z.R.G. and C.L. analyzed the topology. J.C., Z. L., and X.C. carried out the FCC reaction. Z.R.G. and M.A.C. performed the large molecule analysis. Z.R.G., C.L., J.L., and M.A.C. prepared the draft. All the authors discussed the results and revised the manuscript. 
Competing interests: Q.-F.L., Z.R.G., C.L., J.L., and F.-J.C. have filed a patent on zeolite ZEO-1. Z.R.G. and J.L. are affiliated with the company holding the rights on that patent.

Data and materials availability: All data are available in the main text or the supplementary materials. Crystallographic parameters for the structure of as-made and calcined ZEO-1 refined against SPXRD and calcined ZEO-1 refined against cRED data are archived at the Cambridge Crystallographic Data Center (www.ccdc.cam.ac.uk/) under reference nos. CCDC 21127742112776.

\section{Supplementary Materials}

Materials and Methods

Figs. S1 to S22

Tables S1 to S14

References (18-28)

Movie S1

Data S1 to S3 